\title{
Orthogonal and Biorthogonal Wavelet Analysis of Visual Evoked Potentials
}

\author{
Ahmed Fadhil Hassoney \\ Faculty of Physical Science \\ UTM, Johor, Malaysia.
}

\author{
Abd Khamim Ismail \\ Faculty of Physical Science \\ UTM, Johor, Malaysia.
}

\author{
Hentabli Hamza \\ Faculty of Comp. Science and \\ Infor. System, UTM, Johor,
}

\begin{abstract}
In the present work the performance of orthogonal and Biorthogonal wavelet filters were analyzed for visual evoked potentials (VEP) on a variety of noisy signals. The signals were analyzed at different signal to noise ratio (SNR). This research proposed a method for the selection of the best analysis. The proposed method used longest common subsequence (LCS) and basic local alignment search tool (BLAST) to measure the analysis performance objectively and visual quality subjectively of the signal analysis. It was found that orthogonal wavelets outperform the biorthogonal ones in both the criteria especially at high noisy signal.
\end{abstract}

\section{General Terms}

Signal analysis and processing.

\section{Keywords}

Wavelet transforms, longest common subsequence, Basic Local Alignment Search Tool

\section{INTRODUCTION}

The wide application of visual evoked potentials (VEPs) urged the researcher to repeat the same question, how can the picked up signals be improved in order to give a better view to the observer.

Wavelet transform is the newest technique to replace the traditional time-frequency by time-scale signal processing. The major advantage of wavelet is the ability to perform local analysis that is, to analyze a localized area of a larger signal; however time varying non-stationary waveforms are decomposed using wavelet analysis. Neuroelectric waveforms are non-stationary signals and wavelet techniques analyze such signals by providing excellent joint time frequency resolution.

Wavelet analysis had been successfully applied for analysis of EEG potentials and spike detection [2]. Some studies used wavelet transforms with their BCI system based on P300 response [3]. Steady state sweep visual evoked potentials wavelet decomposition and multi-resolution decomposition and denoising of VEP and AEP are correctly done by using biorthogonal wavelets [4][5]. Quiroga et. al. show a good example of wavelet transform in the analysis of the frequency composition of evoked potentials, it showed a better performance of wavelet decomposition as compared to the Fourier based method [6]. Classification of the signals with the help of the wavelet functions have been shown in many studies, likewise the study used second order Gaussian wavelet kernels with multifocal visual evoked potentials (mfVEP) [7]. Daubechies wavelet (db4) was used to distinguish the normal and abnormal VEP responses from each other [8].

VEP signal decomposition and denoising were studied using symlet 5 [9]. Db4 and coif3 were also tested to extract feature of P300 oaaball [10]. Coiflet wavelet was used for classification of EEG for brain computer interface [11].
This present research introduces a comparative study of one dimensional discrete wavelets function between orthogonal (Dubechies (db), Symlet (sym) and Coiflet (cif)) and biorthogonal wavelets of visual evoked potentials to find the best analysis that matches perfectly with the original signal. The basic measure of the performance of the analysis algorithm is the longest common subsequence (LCS) and basic local alignment search tool (BLAST), which are defined by matching the string of the two sequences; the analysis of various wavelet families for signal processing on a variety of signals with additional known noise and then process it to compare the performance of wavelets. According to this analysis, the selection of the best wavelet for VEP signal processing taking into account improvement in the signal to noise ratio (SNR) was shown.

\section{WAVELET FAMILIES}

Wavelet families with filter can be divided into two main categories, orthogonal and Biorthogonal wavelets, which have different properties of basic functions. Orthogonality decorrelates the transform coefficients by minimizing redundancy. Symmetry provides linear phase and minimize border arti-facts. Other Important properties of wavelet functions in signal processing applications are compact support, symmetry, regularity and degree of smoothness [12].

\section{QUALITY MEASURES}

In this research the performances of signal processing techniques are mainly analyzed on the basis of two measures: Longest common subsequence (LCS) and basic local alignment search tool (BLAST). LCS is defined as finding the longest subsequence common to all sequences in a set of sequences. Note that, subsequence is different from a substring and the longest matching subsequence between two strings; the analyzed one as compared to the original. BLAST enables a researcher to compare a query sequence with a library or database of sequences and identify library sequences that resemble the query sequence above a certain threshold. Visual quality of the signal is also considered as subjective quality measures.

\section{EXPERIMENTAL-RESULTS, ANALYSIS AND COMPARISON}

Orthogonal and Biorthogonal wavelet families were analyzed for visual evoked potentials signal and their results were compared. First, transient visual evoked potentials signal (VEP) were simulated based on the standard ISCEV [13], then white Gaussian noise was added to the original signal at five different signal to noise ratio $(0,5,10,-5$, and $-10 \mathrm{db})$. Biorthogonal and orthogonal (Daubchies, symlet and coiflet) wavelet analysis were applied to the noisy signal in order to improve signal to noise ratio and get back the original signal. Figure 1 shows the original signal and signal with additional 
noises. Figure 2 shows orthogonal (Daubchies, symlet and coiflet) and biorthogonal wavelet analysis of the noisy signals. Then longest common subsequence (LCS) and basic local alignment search tool (BLAST) are used as quality measurement of the analysis. The below tables describe these processes. The bold numbers in the tables represent the biggest matches between the analyses

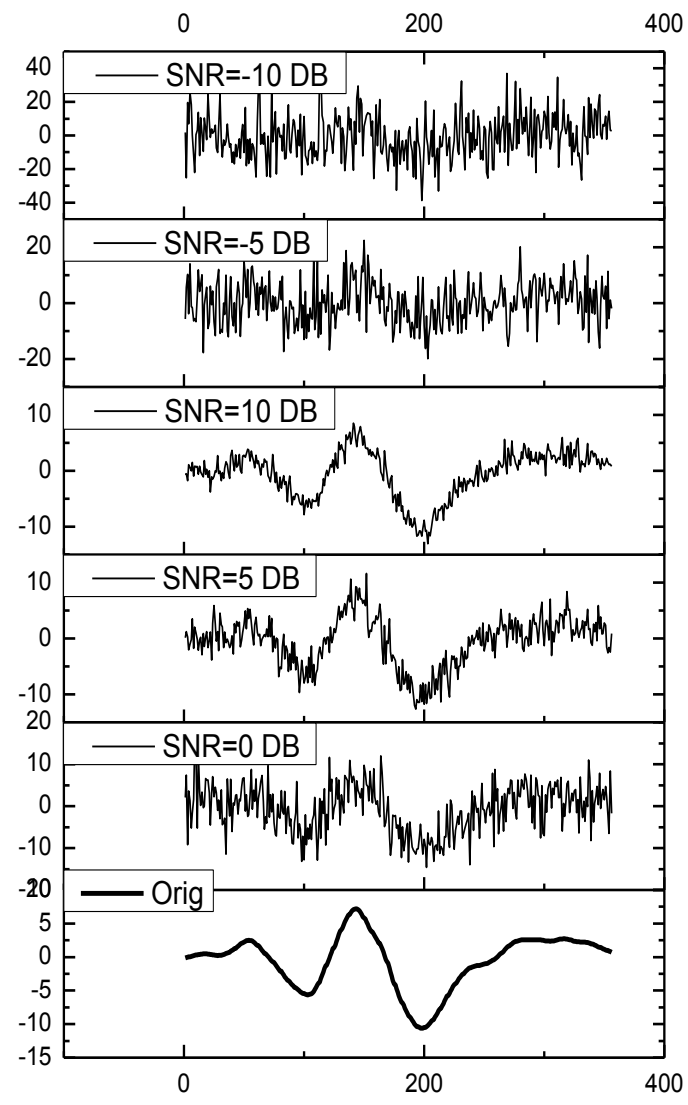

Fig 1: Original signal and signals with additional noise

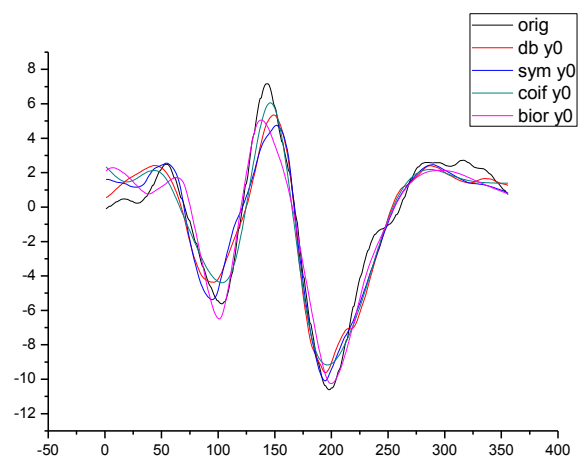

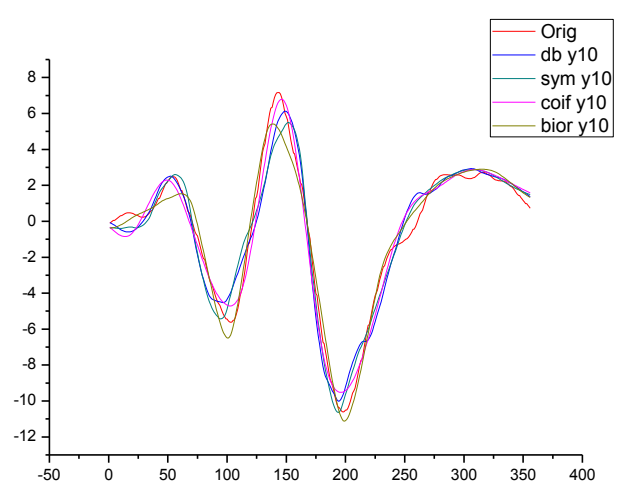
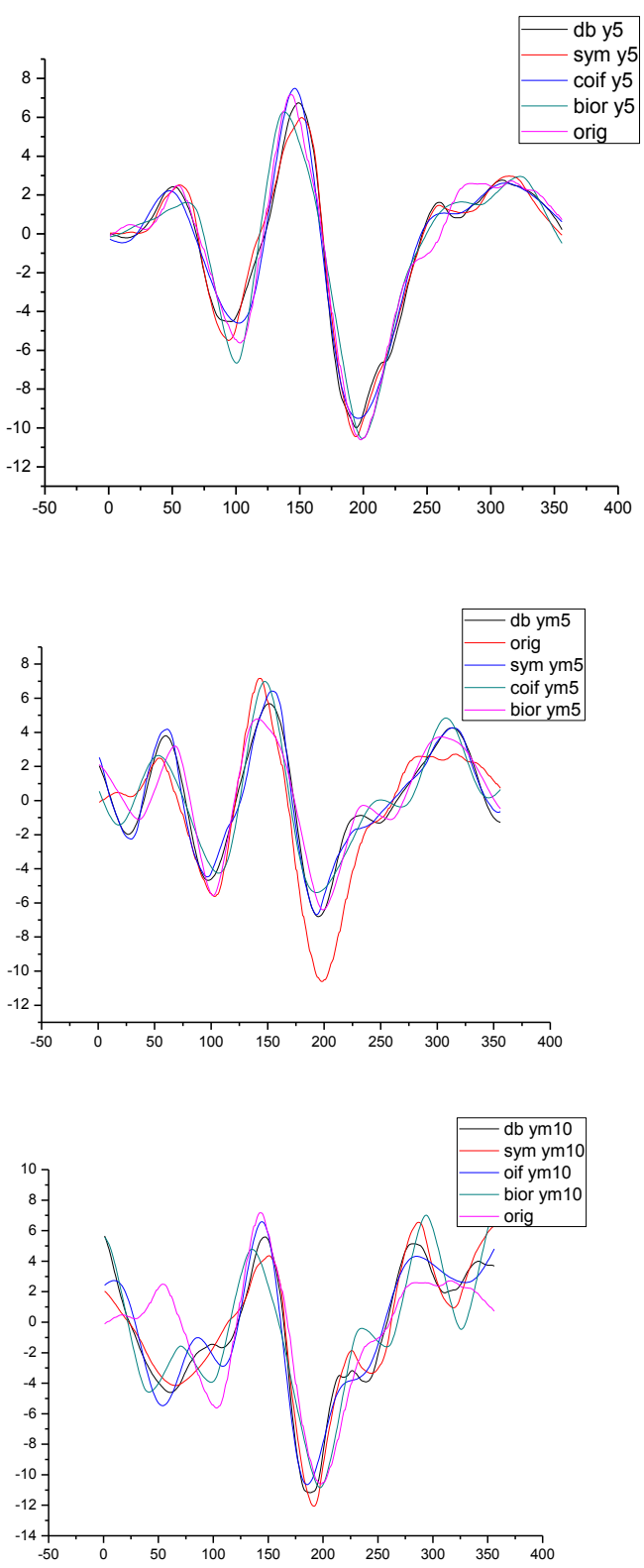

Fig 2: Compression results of orthogonal and biorthogonal wavelet signals analysis, each at decomposition level 5 and order 5 for orthogonal and 5.5 for biorthogonal wavelet. 
Table 1. Longest common subsequence (LCS) of wavelet analysis

\begin{tabular}{|c|c|c|c|c|}
\hline \multicolumn{4}{|c|}{ Longest common subsequence (LCS) } \\
\hline \multirow{2}{*}{} & \multirow{2}{*}{ Bior } & \multicolumn{3}{|c|}{ ortho } \\
\cline { 3 - 5 } & & Db & sym & coif \\
\hline RMS=0 & 49.72 & 48.9 & $\mathbf{4 9 . 8 6}$ & 49.72 \\
\hline RMS=5 & 49.86 & 50.13 & 50 & $\mathbf{5 0 . 2 7}$ \\
\hline RMS=10 & 48.9 & $\mathbf{5 0 . 2 7}$ & 49.72 & 50 \\
\hline RMS=-5 & 50.27 & 50 & 50.27 & $\mathbf{5 0 . 2 7 7}$ \\
\hline RMS=-10 & 49.72 & 50 & 49.44 & $\mathbf{5 0}$ \\
\hline
\end{tabular}

Table 2. Basic Local Alignment Search Tool (BLAST) of wavelet analysis

Basic Local Alignment Search Tool (BLAST)

\begin{tabular}{|c|c|c|c|c|}
\hline \multirow{2}{*}{} & \multirow{2}{*}{ Bior } & \multicolumn{3}{|c|}{ ortho } \\
\cline { 3 - 5 } & & Db & sym & coif \\
\hline RMS=0 & 6.42 & 7.55 & $\mathbf{1 0 . 7 3}$ & 3.18 \\
\hline RMS=5 & 8.2 & $\mathbf{1 7 . 4 9}$ & 6.9 & 13.92 \\
\hline RMS=10 & $\mathbf{1 6 . 7 2}$ & 14.1 & 16.33 & 13.55 \\
\hline RMS=-5 & 3.48 & 3.18 & 4.09 & $\mathbf{8 . 0 4}$ \\
\hline RMS=-10 & 2.59 & 2.89 & 2.74 & $\mathbf{3 . 6 3}$ \\
\hline
\end{tabular}

From these experiments and trials it can be deduced that the best order to VEP analysis equals to 5 , the decomposition level was also 5; whereas decomposition at a higher level will disturb the signal instead of adjusting it. This research deduced that orthogonal wavelet function gives the better analysis performance as compared to biorthogonal wavelet families, considered in this experiment. Also the coiflet5 wavelet shows the best analysis in both quality measurements (LCS and BLAST) at negative signal to noise ratio. This is due to the reason of orthogonal wavelets use the filters with the same order $(\mathrm{N})$, only one order for both decomposition and reconstruction filters and also the signal processing is about one dimensional signal. This is differing from Image processing, the preferable family of wavelet is biorthogonal, that can use two filters with similar or dissimilar order for decomposition and reconstruction [12]. Accordingly, this leads to different wavelet properties. Moreover, it is also observed that the BIOR wavelet families take much more computational time in comparison to other wavelet families considered in our experiment. Also, it was found that as the filter order increases in a given wavelet family, the analysis performance increases, but the visual quality of the signal becomes poorer. The higher order of filters involves the longer filters, which involves unclear signals.

\section{CONCLUSION}

This study presents an analysis and comparison of the wavelet families for VEP signal analysis considering LCS and BLAST quality measure of the signal as compared to the standard. The effects of Biorthogonl, Daubechies, Coiflet and Symlet wavelet families on simulated signals are examined. The results for a wide range of wavelet families were analyzed and it was found that the wavelet coiflet5 provides the best analysis performance for VEP analysis, especially for high noisy signals. The proposed techniques LCS and BLASET provide a good tool to test the biggest match between the standard signal and the signals after analysis.

\section{ACKNOWLEDGMENTS}

The authors would like to thank the department of physics, faculty of science Universiti Teknologi Malaysia for their support.

\section{REFERENCES}

[1] Aoyagi, M. and Harada, J. 1988. Application of fast Fourier transform to auditory evoked brainstem response. Informatics for Health and Social Care, 13(3), 211-220.

[2] Kalayci, T. and Özdamar, Ö. 1995. Wavelet processing for automated neural network detection of EEG spikes. IEEE Engineering in Medicine and Biology Magazine, 14(2), 160-166.

[3] Qiao, X. and Yan, M. 2011. P300 Feature Extraction for Visual Evoked EEG Based on Wavelet Transform. AICI Springer-Verlag Berlin Heidelberg, 7003: 561-567.

[4] Weiderpass, H. A., Yamamoto, J. F., Salomao, S. R., Berezovsky, A., Pereira, J. M., Sacai, P. Y., Oliveira, J. P., Costa, M. A. and Burattini, M. N. 2008. Steady-state sweep visual evoked potential processing denoised by wavelet transform. International Society for Optical Engineering, 6917: 69171A.

[5] Quiroga, R. Q. 2000. Obtaining single stimulus evoked potentials with wavelet denoising. Physica D: Nonlinear Phenomena, 145(3-4), 278-292.

[6] Quiroga, R. Q., Sakowitz, O. W., Basar, E. and Schürmann, M. 2001. Wavelet Transform in the analysis of the frequency composition of evoked potentials. Brain Research Protocols, 8(1), 16-24.

[7] Thie, J., Sriram, P., Klistorner, A. and Graham, S. L. 2012. Gaussian wavelet transform and classifier to reliably estimate latency of multifocal visual evoked potentials (mfVEP). Vision Research 52(1), 79-87.

[8] Akbari, M. and Azmi, R. 2011. Automatic Classification of Visual Evoked Potentials Based on Wavelet Analysis and Support Vector Machine. 6th International Advanced Technologies Symposium (IATS'11) Elazı̆̆, Turkey 227-230.

[9] Samar, V. J., Bopardikar, A., Rao, R. and Swartz, K. 1999. Wavelet Analysis of Neuroelectric Waveforms: A Conceptual Tutorial. Brain and Language, 66(1), 7-60.

[10] Gupta, C. N., Khan, Y. U., Palaniappan, R. and Sepulveda, F. 2009. Wavelet Framework for Improved Target Detection in Oddball Paradigms Using P300 and Gamma Band Analysis. Biomedical Soft Computing and Human Sciences, 14(2): 61-67.

[11] Yong, P. A., Hurley, N. J. and Silvestre, G. C. M. 2005. Single-trial EEG classification for brain-computer interface using wavelet decomposition. In European Signal Processing Conference, EUSIPCO.

[12] Kumari, S. and Vijay, R. 2011. Analysis of orthogonal and biorthogonal wavelet filters for image compression. International journal of computer applications, 21(5), 1719.

[13] Odom, J. V., Bach, M., Brigell, M. E., Holder, J. E., McCulloch, D. L., Tormene, A. P. and Vaegan. ISCEV standard for clinical visual evoked potentials (2009 update). Doc Ophthalmol, 120(1), 111-119. 